\title{
Multi Target Direction-of-Arrival Tracking using Road Priors
}

\author{
Volkan Cevher, Rajbabu Velmurugan, and James H. McClellan \\ School of Electrical and Computer Engineering \\ Georgia Institute of Technology \\ 777 Atlantic Drive \\ Atlanta, GA 30332-0250, USA \\ 404-387-0809 \\ \{volkan, rajbabu,jim.mcclellan\}@ece.gatech.edu
}

Abstract-In this paper, we present a multi target particle filter DOA tracker that can incorporate road prior information at a single array node. The filter uses a batch of DOA's to determine the state vector, based on an image template matching idea. The filter likelihood is derived with the joint probability density association principles so that no DOA measurement is associated to more than one target. The filter state update has the target DOA, the target velocity over range ratio, and the target heading parameters. We present two approaches for incorporating the road information. In the first approach, the road prior is injected at the weighting stage of the tracker, where a raised mixture Gaussian distribution, derived from the road headings at the target DOA, constraints the particles. The second approach is based on modifying the state update function with a compound model, where a mixture of the constant velocity model and the road information is used. In this case, the filter uses an online EM algorithm to update the state vector along with the mixture components. Computer simulations demonstrate the performance of the approaches.

\section{TABLE OF CONTENTS}

\section{INTRODUCTION \\ 2 PARTICLE Filter \\ 3 THE ROAD PRIOR INFORMATION \\ 4 Simulations \\ 5 CONCLUSIONS}

\section{INTRODUCTION}

In target tracking problems, constrained target paths along known roads, due to terrain conditions or obstructions, present an opportunity for improved tracking [1-6]. In the literature, this problem is considered, using state spaces that employ spatial parameters that are naturally linked with the constraints. It is, however, harder to incorporate the road information to a direction-of-arrival (DOA) tracker, because the spatial position constraints do not translate well into regular angle constraints.

Prepared through collaborative participation in the Advanced Sensors Consortium sponsored by the U. S. Army Research Laboratory under the Collaborative Technology Alliance Program, Cooperative Agreement DAAD19-0102-0008.

$0-7803-9546-8 / 06 / \$ 20.00 / \odot 2006$ IEEE

IEEE AC paper \#1265
It is a challenging signal processing problem to track the bearing angles of multiple targets using an acoustic array in the presence of noise or interferers [7-11]. To formulate the DOA tracking problem using state space models [12-14], we need an observation equation that relates the state vector (i.e., target DOA's and possibly motion states) to the acoustic microphone outputs, and a state update equation that constrains the dynamic nature of the state vector. In most cases, it is important to use nonlinear and non-Gaussian state-space models despite their computational complexity [15].

In this paper, we first summarize a particle filter algorithm to track the DOA's of multiple maneuvering targets, using an acoustic node, which contains an array of microphones with known positions [16]. The filter state consists of the DOA $\theta_{k}(t)$, the heading direction $\phi_{k}(t)$, and the logarithm of velocity over range $Q_{k}(t)=\log \left(v_{k} / r_{k}(t)\right)$ for each target $k$. A partitioning approach is used to create the multiple target state vector, where each partition is assumed to be independent; however, the filter observation likelihood uses a joint probability assignment of each partition similar to the JPDA method [17].

We show two ways to incorporate the road information into the DOA tracker. In the first approach, the weighting method, the road information is treated as a pseudo-measurement and appears at the weighting stage of the algorithm. The pseudo measurement idea has been explored in [5] for particle filters. Our approach differs from [5], because (i) our filter proposal function uses an approximation of the full tracking posterior without using the road information, and (ii) our pseudo measurement likelihood allows the targets to come-off the road. The second approach, the mixture method, uses a mixture model for the target state update function by combining the motion equations with the road information. By adaptively calculating the mixture probabilities by an EM algorithm $[18,19]$, the particle filter outputs improved state vector estimates along with the probability that the target is following a certain road.

The particle filter uses a batch of DOA's to determine the state vector, based on an image template matching idea. In our problem, a DOA image is first formed, when a batch of DOA observations are received. Then, image templates for target tracks are created using the state update function 
and the target state vectors. By determining the best matching template (e.g., probable target track) to the image, the target state-vectors are estimated. Because the observations are treated as an image, the data association and DOA ordering problems are naturally alleviated. Moreover, by assuming that the DOA observations are approximately normally distributed around the true target DOA tracks, with constant DOA miss-probability and clutter density, a robust particle filter tracker is formulated.

The presented particle filter uses a front-end processor, i.e., a frequency-adaptive acoustic beamformer, producing sufficient statistics for state vector with known statistical properties: $M$-DOA estimates during a batch size of $T$ seconds ( $M>3$ for each target for observability of the state $[9,13])$. Hence, the target signal assumptions (e.g., wideband or narrow-band target signals) are handled by the front-end processor. Therefore, the probability density functions in the particle filter mechanics are independent of the acoustic data, conditioned on the sufficient statistics. Moreover, since the filter is built on state sufficient statistics, which compresses the data to be processed for tracking [20], it achieves a significant reduction in computational requirements.

The particle filter importance function proposes particles for each target partition independently to increase the efficiency of the algorithm. Because the ground targets can wander off the road, we do not directly use the road information while proposing the particles. To derive the partition proposal function, we use the Laplace's method to approximate each partition posterior by a Gaussian around its mode [14,21,22]. We calculate the partition modes using a robust Newton-Raphson search method that imposes smoothness constraints on the target motion.

The organization of the paper is as follows. Section 2 gives the details of the multi target DOA particle filter tracker. Section 3 explains the weighting and mixture methods for incorporating the road prior information into the angle-only tracking particle filter. The performance of the methods are demonstrated on simulated data in Sect. 4.

\section{PARTICLE Filter}

\section{State Equation}

The filter state vector $\mathbf{x}_{t}=\left[x_{1}^{T}(t), x_{2}^{T}(t), \ldots, x_{K}^{T}(t)\right]^{T}$ consists of the concatenation of partitions $x_{k}(t)$ for each target, indexed by $k$, where $K$ is the number of targets at time $t$. Each partition has the corresponding target motion parameters $x_{k}(t) \triangleq\left[\theta_{k}(t), Q_{k}(t), \phi_{k}(t)\right]^{T}$, where the $k^{\text {th }}$ target DOA is $\theta_{k}(t)$, the heading direction is $\phi_{k}(t)$, and the logarithm of the velocity-range ratio is $Q_{k}(t)$. The angle parameters $\theta_{k}(t)$ and $\phi_{k}(t)$ are measured counterclockwise with respect to the $x$-axis.

The state update equation can be derived from the geometry imposed by the locally constant velocity model. The resulting state update equation is nonlinear:

$$
x_{k}(t+T)=h_{T}\left(x_{k}(t)\right)+u_{k}(t),
$$

where $u_{k}(t) \sim \mathcal{N}\left(0, \Sigma_{u}\right)$ with $\Sigma_{u}=\operatorname{diag}\left\{\sigma_{\theta, k}^{2}, \sigma_{Q, k}^{2}, \sigma_{\phi, k}^{2}\right\}$ and $h_{T}\left(x_{k}(t)\right)=$

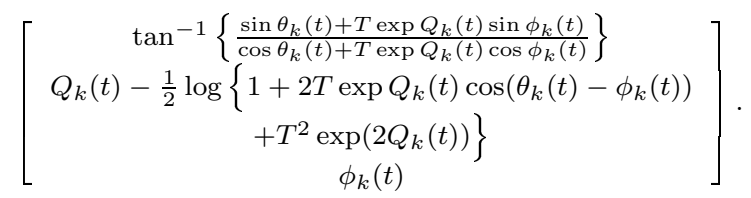

The analytical derivations of (2) can be found in $[9,10]$. The reference [10] also discusses state update equations based on constant acceleration assumption. Hence, the constant velocity state update density function is the following Gaussian distribution $p\left(x_{k}(t+T) \mid x_{k}(t)\right)=$

$$
f_{1}\left(x_{k}(t+T) \mid x_{k}(t)\right)=\mathcal{N}\left(h_{T}\left(x_{k}(t)\right), \Sigma_{u}\right),
$$

where $\mathcal{N}(\mu, \Sigma)$ denotes the normal distribution with mean $\mu$ and covariance $\Sigma$.

\section{Observation Equation}

The observations $\mathbf{y}_{t, f}=\left\{y_{t+m \tau, f}(p)\right\}_{m=0}^{M-1}$ consist of all the batch DOA estimates from the beamformer block indexed by $m$. Hence, the acoustic data of length $T$ is segmented into $M$ segments of length $\tau$. These segments are processed by a beamformer based on the temporal frequency structure of the signals to calculate possible DOA estimates. This procedure may be repeated $F$ times for narrow-band signals at each frequency indexed by $f$. Note that only the peak locations are kept in the beamformer power pattern. Moreover, the peak values, indexed by $p$, need not be ordered or associated with the previous time in the batch and the number of peaks retained can be time-dependent.

The batch of DOA's, $\mathbf{y}_{t, f}$, is assumed to form an approximately normally distributed cloud around the true target DOA tracks (refer to Fig. 1 for visualizing the observation model for one frequency). In addition, only one DOA is present for each target at each $f$ or the target is missed: multiple DOA measurements imply presence of clutter or other targets. We also assume that there is a constant detection probability for each target denoted by $\kappa^{f}$, where dependence on $f$ is allowed.

The particle filter observation model also includes a clutter model, because beamformers can produce spurious DOA peaks as output (e.g., the sidelobes in the power vs. angle patterns) [23]. To derive the clutter model, it is assumed that the spurious DOA peaks are random with uniform spatial distribution on the angle space, and are temporally as well as spatially independent. In this case, the probability distribution for the number of spurious peaks is best approximated by the Poisson distribution with a spatial density [17,24]. We use the following pdf for the spurious peaks:

$$
p(\theta \mid \theta \text { is spurious })=\lambda \text {, }
$$




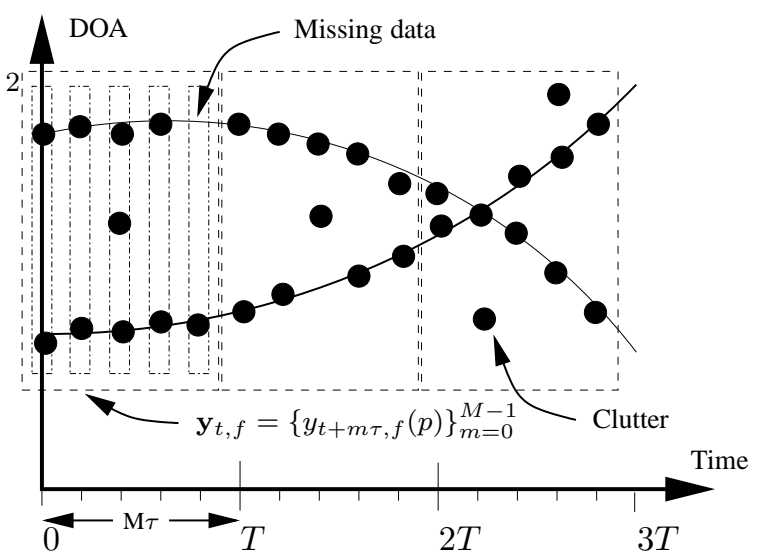

Figure 1. The circles are the DOA estimates, calculated at a single frequency, using the acoustic data received during a period of length $\tau$. In this example, the maximum number of beamformer peaks $P$ is 3 . Given the observations $\mathbf{y}_{t, f}$, the objective of the particle filter is to determine the state $x_{k}(t)$ for the two targets, which completely parameterizes the solid curves.

where $\lambda$ is the clutter density parameter.

We now derive the data-likelihood function using the joint probabilistic data association arguments found in [17]. Similar arguments for active contour tracking that is relevant to this paper are found in [25]. Consider the output of one batch period $\mathbf{y}_{m, f}=y_{t+m \tau, f}(p)$, where $p=0,1, \ldots, P_{m, f}$ for each $f$ and $m$. The DOA's $\mathbf{y}_{m, f}$ may belong to none, or some combination, or all of the targets in the particle filter partitions. Hence, we first define a notation to represent possible combinations between the data and the particle filter partitions to effectively derive the observation density.

Define a set $\mathcal{I}_{n}$ that consists of $n$-unordered combination of all $K$-partitions of the particle filter state vector: $\mathcal{I}_{n} \in\left\{{ }_{K} \mathcal{C}_{n}\right\}$, where ${ }_{K} \mathcal{C}_{n}$ is number of ways of picking $n$-unordered outcomes from $K$ possibilities. Each element of $\mathcal{I}_{n}$ has $n$ numbers, and there are a total of ${ }_{K} \mathcal{C}_{n}$ elements. For example, when $K=3$ and $n=2$, then $\mathcal{I}_{2}=\{\{1,2\},\{1,3\},\{2,3\}\}$, each element referring to subset of the individual partitions of the particle state vector. We refer to the individual elements of this set using the notation $\mathcal{I}_{n}(j)$, where $j=1, \ldots,{ }_{K} \mathcal{C}_{n}$. Hence, $\mathcal{I}_{2}(2)=\{1,3\}$. Then, denote ${ }_{n} \mathbf{x}_{t}(j) \in\left\{x_{i}(t) \mid i \in \mathcal{I}_{n}(j), x_{i}(t) \in \mathbf{x}_{t}\right\}$ as a single realization from the set $\mathcal{I}_{n}$. Using the same example, ${ }_{2} \mathbf{x}_{t}(3)=\left[x_{2}^{T}(t), x_{3}^{T}(t)\right]^{T}=\left[\hat{x}_{1}^{T}(t), \hat{x}_{2}^{T}(t)\right]^{T}$. The elements of the vector ${ }_{n} \mathbf{x}_{t}(j)$ are shown, in order, by $\hat{x}_{i}(t)$ $(i=1, \ldots, n)$, given the parameters $n$ and $j$.

We denote $\pi_{n, j}\left(\mathbf{y}_{m, f}\right)=p\left(\left.\mathbf{y}_{m, f}\right|_{n} \mathbf{x}_{t}(j)\right)$ as the probability density function of the data, where only $n$-DOA's belong to the targets defined by the partitions of ${ }_{n} \mathbf{x}_{t}(j)$. Hence, when $n=0$, all data is due to clutter:

$$
\pi_{0,1}\left(\mathbf{y}_{m, f}\right)=\lambda^{P_{m, f}}
$$

The probability density $\pi_{n, j}\left(\mathbf{y}_{m, f}\right)$ can be calculated by noting that (i) there are $P_{m, f} ! /\left(P_{m, f}-n\right)$ ! ordered ways of choosing DOA's to associate with the $n$-subset partitions, and (ii) the remaining $\left(P_{m, f}-n\right)$-DOA's are explained by the clutter. Therefore,

$$
\begin{aligned}
& \pi_{n, j}\left(\mathbf{y}_{m, f}\right)= \frac{\left(P_{m, f}-n\right) ! \lambda^{P_{m, f}-n}}{P_{m, f} !} \times \\
& \sum_{p_{1} \neq p_{2} \neq \ldots \neq p_{n}}^{P_{m, f}} \prod_{i=1}^{n} \psi_{t, m, f}\left(p_{i} \mid \hat{x}_{i}\right),
\end{aligned}
$$

where the function $\psi$ is derived from the assumption that the associated target DOA's form a Gaussian distribution around the true target DOA tracks:

$$
\begin{aligned}
\psi_{t, m, f}\left(p_{i} \mid x_{i}\right)= & \frac{1}{\sqrt{2 \pi \sigma_{\theta}^{2}(m, f)}} \times \\
& \exp \left\{-\frac{\left(h_{m \tau}^{\theta}\left(x_{i}(t)\right)-y_{t+m \tau, f}\left(p_{i}\right)\right)^{2}}{2 \sigma_{\theta}^{2}(m, f)}\right\},
\end{aligned}
$$

where the superscript $\theta$ on the state update function $h$ refers only to the DOA component of the state update and $\sigma_{\theta}^{2}(m, f)$ is supplied by a beamformer, by using the curvature of the DOA estimate at the power vs. angle pattern [23].

Note that the DOA distribution (7) is not a proper circular distribution for an angle space. For angle spaces, the von Mises distribution is used as a natural distribution [26]. The von Mises distribution has a concentration parameter with a corresponding circular variance. It can be shown that for small $\sigma_{\theta}^{2}<<1$ (high concentration), the von Mises distribution tends to the Gaussian distribution in (7) [27]. Because the von Mises distribution has numerical issues for small DOA variances, the Gaussian approximation (7) is used in this paper. Hence, special care must be taken in the implementation to handle angle wrapping issues.

The Gaussian in (6) $\psi(\cdot \mid \cdot)$ are directly multiplied, because the partitions are assumed to be independent. To elaborate, consider $n=2$ and $j=3$ from the example of $\mathcal{I}_{2}$ above:

$$
\begin{aligned}
\pi_{2,3}\left(\mathbf{y}_{m, f}\right) & \propto \sum_{p_{1}=1}^{P_{m, f}} \sum_{p_{2}=1, p_{1} \neq p_{2}}^{P_{m, f}} \psi_{t, m, f}\left(p_{1} \mid \hat{x}_{1}\right) \psi_{t, m, f}\left(p_{2} \mid \hat{x}_{2}\right) \\
& \propto \sum_{p_{1}=1}^{P_{m, f}} \sum_{p_{2}=1, p_{1} \neq p_{2}}^{P_{m, f}} \psi_{t, m, f}\left(p_{1} \mid x_{2}\right) \psi_{t, m, f}\left(p_{2} \mid x_{3}\right) .
\end{aligned}
$$

Hence, the density $\pi_{2,3}\left(\mathbf{y}_{m, f}\right)$ is a Gaussian mixture that peaks, when the updated DOA components of the partitions 2 and $3\left(h_{m \tau}^{\theta}(\cdot)\right)$ are simultaneously close to the observed DOA's. Note that (8) guarantees that no measurement is assigned to multiple targets simultaneously.

Given the densities $\pi_{n, j}$, the observation density function can be constructed as a combination of all the target association 
hypotheses. Hence, by adding mixtures that consist of the data permutations and the partition combinations, we derive the observation density:

$$
p\left(\mathbf{y}_{t} \mid \mathbf{x}_{t}\right)=\prod_{f=1}^{F} \prod_{m=0}^{M-1} \sum_{n=0}^{K} \frac{\kappa_{n, K}^{f}}{{ }_{K} \mathcal{C}_{n}} \sum_{j=1}^{K \mathcal{C}_{n}} \pi_{n, j}\left(\mathbf{y}_{m, f}\right)
$$

In (9), the parameters $\kappa_{n, K}^{f}\left(\sum_{n} \kappa_{n, K}^{f}=1\right)$ are the elements of a detection (or confusion) matrix. For example, when $K=2, \kappa_{0,2}^{f}$ is the probability that no target DOA is in the beamformer output, whereas $\kappa_{1,2}^{f}\left(\kappa_{2,2}^{f}\right)$ means that 1 (2) target DOA('s) are present in the beamformer output at each $f$. These fixed values have to be provided by the user. However, they should be changed adaptively to improve robustness of the particle filter output. For example, when two partitions $k_{1}$ and $k_{2}$ have close DOA tracks and are about to cross, it is possible that the beamformer's Rayleigh resolution is not enough to output two DOA's for both targets. Then, we change the confusion matrix to indicate the possibility that one of the targets will likely be missed.

\section{Particle Filter Proposal Function}

In the problem of DOA-only multiple target tracking, the proposal function poses difficult challenges because (i) the state vector dimension is proportional to the number of targets $K$, hence the number of particles to represent posterior can increase significantly as $K$ increases (the curse of dimensionality), (ii) in many cases, the targets maneuver, hence full posterior approximations are required for robust tracking, and (iii) for full posterior approximations, robustly determining the DOA-only data-likelihood is rather hard. We address each of these challenges in this section. Note that the proposal function of the filter does not incorporate the road information. This is because we allow the targets to come off the road. If a target is passing a bridge, where the constraint completely determines the target motion, then to tackle this scenario, we change the probability that the target is on the road to one.

Partitioned Sampling - A partitioned sampling approach is used to reduce the curse of dimensionality in the particle filter. The basic idea is as follows. Suppose that we incorrectly factor the tracking posterior density as

$$
\begin{aligned}
p\left(\mathbf{x}_{t} \mid \mathbf{y}_{t}, \mathbf{x}_{t-T}\right) & \propto p\left(\mathbf{y}_{t} \mid \mathbf{x}_{t}\right) p\left(\mathbf{x}_{t} \mid \mathbf{x}_{t-T}\right) \\
& =\prod_{k=1}^{K} p\left(\mathbf{y}_{t} \mid x_{k}(t)\right) \prod_{k=1}^{K} p\left(x_{k}(t) \mid x_{k}(t-T)\right) \\
& =\prod_{k=1}^{K} p\left(\mathbf{y}_{t} \mid x_{k}(t)\right) p\left(x_{k}(t) \mid x_{k}(t-T)\right) \\
& \propto \prod_{k=1}^{K} q_{k}\left(x_{k}(t) \mid \mathbf{y}_{t}, x_{k}(t-T)\right) .
\end{aligned}
$$

In this case, the target posterior is conveniently a product of the partition posteriors $q_{k}(\cdot \mid \cdot)$. We can then generate samples for each partition according to its posterior (i.e., $x_{k}^{(i)} \sim$ $\left.q_{k}\left(x_{k}(t) \mid \mathbf{y}_{t}, x_{k}(t-T)\right)\right)$ and merge them to represent $\mathbf{x}_{t}$. It can be proved that the resulting particle distribution is the same as when we generate $\mathbf{x}_{t}$ directly from the full posterior $p\left(\mathbf{x}_{t} \mid \mathbf{y}_{t}, \mathbf{x}_{t-T}\right)$. However, in the partitioned sampling case, the computational complexity of the state vector generation is linear with respect to the number of targets $K$ as opposed to exponential when the state vector is sampled from the full posterior.

In our problem, we can approximately factor out the tracking posterior to exploit the computational advantage of the partitioned sampling. Note that in our case, the target dynamics can already be factored out because we assume the targets are moving independently. ${ }^{1}$ Unfortunately, the observation density does not factor out, because the observed DOA data cannot be immediately associated with any of the partitions. However, for a given partition, if we assume that the data is only due to that partition and clutter (hence, the DOA data corresponding to other partitions are treated as clutter), we can do the following approximate factorization on the observation likelihood (9):

$$
\begin{aligned}
p\left(\mathbf{y}_{t} \mid \mathbf{x}_{t}\right) & \approx \prod_{k=1}^{K} p\left(\mathbf{y}_{t} \mid x_{k}(t)\right) \\
& =\prod_{k=1}^{K} \prod_{f=1}^{F} \prod_{m=0}^{M-1}\left\{\kappa_{0,1}^{f} \lambda^{P_{m, f}}\right. \\
& \left.+\kappa_{1,1}^{f} \lambda^{P_{m, f}-1} \sum_{p=1}^{P_{m, f}} \frac{\psi_{t, m, f}\left(p \mid x_{k}\right)}{P_{m, f}}\right\} .
\end{aligned}
$$

Hence, for our problem, each partition posterior is given by $q_{k}\left(x_{k}(t) \mid \mathbf{y}_{t}, x_{k}(t-T)\right) \propto p\left(\mathbf{y}_{t} \mid x_{k}(t)\right) p\left(x_{k}(t) \mid x_{k}(t-T)\right)$,

where $p\left(\mathbf{y}_{t} \mid x_{k}(t)\right)$ is given in (11) and $p\left(x_{k}(t) \mid x_{k}(t-T)\right)=$ $f_{1}\left(x_{k}(t) \mid x_{k}(t-T)\right)$ as in (3). Note that (11) will not be used as the data-likelihood of the particle filter. The above approximate factorization of the data-likelihood is to make use of the partitioned sampling strategy to propose particles. To calculate the particle filter weights, the full posterior uses the observation density (9).

A Gaussian Approximation for Partition Posteriors-To capture target maneuvers effectively, we use the current observed data to propose the filter's particle support. The filter uses Laplace's method to approximate $p\left(\mathbf{y}_{t} \mid x_{k}(t)\right)$ in (12) and thereby derive the partition proposal functions of the particle filter, denoted as $g_{k}\left(x_{k}(t) \mid \mathbf{y}_{t}, x_{k}(t-T)\right)$. Laplace's method is an analytical approximation of probability density functions based on a Gaussian approximation of the density around its mode, where the inverse Hessian of the logarithm

\footnotetext{
${ }^{1}$ When the targets are moving closely in tandem, there is a possibility that a beamformer may not resolve them. Hence, they can be treated as a single target. In other cases, the independence assumption still works. However, if high resolution observations (e.g, top down video images of the target plane) are available, it is better to also model the interactions of targets. A good example using Monté-Carlo Markov chain methods can be found in [28].
} 
of the density is used as a covariance approximation [22]. It can provide adequate approximations to posteriors that are as accurate and sometimes more accurate than the approximations based on third-order expansions of the density functions [21]. The computational advantage of this approach is rather attractive, because it only requires first and second order derivatives. The condition for the accurate approximation is that the posterior be a unimodal density or be dominated by a single mode. Hence, it is appropriate for approximating the partition posteriors of the particle filter.

Laplace's approximation requires the calculation of the data statistics. The Laplacian approximation is described in [29] and is implemented with the Newton-Raphson recursion with backtracking for computational efficiency [30,31]. The final expression for the partition proposal functions to be used in the particle filter is given by

$$
g_{k}\left(x_{k}(t) \mid \mathbf{y}_{t}, x_{k}(t-T)\right) \sim \mathcal{N}\left(\mu_{g}(k), \Sigma_{g}(k)\right)
$$

where the Gaussian density parameters are

$$
\begin{aligned}
\Sigma_{g}(k) & =\left(\Sigma_{y}^{-1}(k)+\Sigma_{u}^{-1}\right)^{-1} \\
\mu_{g}(k) & =\Sigma_{g}(k)\left(\Sigma_{y}^{-1}(k) x_{k, \text { mode }}+\Sigma_{u}^{-1} h_{T}\left(x_{k}(t-T)\right)\right),
\end{aligned}
$$

where $x_{k \text {,mode }}$ is the mode of $p\left(\mathbf{y}_{t} \mid x_{k}(t)\right)$, and $\Sigma_{y}^{-1}(k)$ is the Hessian of $p\left(\mathbf{y}_{t} \mid x_{k}(t)\right)$ at $x_{k, \text { mode }}$, calculated by imposing smoothness constraints on the target motion [29]. Pseudocode of the particle filter multi target DOA tracking algorithm is given in Table 1 .

\section{Table 1. Particle Filter Tracker Pseudo-Code}

Given the observed data $\mathbf{y}_{t, f}=\left\{y_{t+m \tau, f}(p)\right\}_{m=0}^{M-1}$ in $[t, t+T)$, do

1. For $i=1,2, \ldots, N$

- For $k=1,2, \ldots, K$

$$
\text { sample } x_{k}^{(i)}(t) \sim g_{k}\left(x_{k}^{(i)}(t) \mid \mathbf{y}_{t}, x_{k}^{(i)}(t-T)\right) \text {, given by (13). }
$$

- Form $\mathbf{x}_{t}^{(i)}=\left[x_{1}^{(i)}(t), x_{2}^{(i)}(t), \ldots, x_{K}^{(i)}(t)\right]^{T}$.

2. Calculate the weights

$$
w_{t}^{*(i)}=w_{t-T}^{(i)} \frac{p\left(\mathbf{y}_{t} \mid \mathbf{x}_{t}^{(i)}\right) p\left(\mathbf{x}_{t}^{(i)} \mid \mathbf{x}_{t-T}\right)}{\prod_{k} g_{k}\left(x_{k}^{(i)}(t) \mid \mathbf{y}_{t}, x_{k}^{(i)}(t-T)\right)},
$$

where $p\left(\mathbf{y}_{t} \mid \mathbf{x}_{t}^{(i)}\right)$ is fully joint observation density, given by (9). 3. Normalize the weights:

$$
w_{t}^{(i)}=\frac{w_{t}^{*}(i)}{\sum_{i} w_{t}^{*}{ }_{t}^{(i)}} .
$$

4. Make estimation: $E\left\{f\left(\mathbf{x}_{t}\right)\right\}=\sum_{i=1}^{N} w_{t}^{(i)} f\left(\mathbf{x}_{t}^{(i)}\right)$.

5. Resample the particles.

\section{THE ROAD PRIOR INFORMATION}

\section{The Weighting Method}

If the road information is available along with the calibrated acoustic node, it is optimal to formulate a new tracker, using the target position and velocity as the state vector. However, it is also possible to incorporate the road information to the DOA-only particle filter tracker, without changing any filter equations. Note that if a target is following a road, its heading direction, in effect, coincides with the road's orientation. Hence, at any given time, as long as the target is on the road, its heading direction will be approximately Gaussian distributed with a mean angle of the road's orientation and some variance. Denote

$$
\begin{aligned}
p_{\{\theta, \operatorname{road}\}}(\phi) & =q+(1-q) \times \\
& \sum_{j} \gamma_{j}\left\{\delta_{\phi} \mathcal{N}\left(\phi_{\{\theta, \operatorname{road}(j)\}}, \sigma_{\phi, \operatorname{road}(j)}^{2}\right)\right. \\
& \left.+\left(1-\delta_{\phi}\right) \mathcal{N}\left(\pi-\phi_{\{\theta, \operatorname{road}(j)\}}, \sigma_{\phi, \operatorname{road}(j)}^{2}\right)\right\}
\end{aligned}
$$

as the heading prior, calculated using (i) the track information, (ii) the acoustic node position, and (iii) the current DOA $\theta$. The parameter $q$ is the probability that a target is not on the road, whereas $\gamma_{j}$ is the probability that a target is on a specific road branch $j$. The heading variance $\sigma_{\phi, \operatorname{road}(j)}^{2}$ limits how much variation is allowed from the road's heading direction. If it is too small, the state estimates will closely follow the road. However, when the targets leave the roads, the filter heading particle distribution may under-represent the actual posterior, deteriorating the estimates until the filter converges back on the target. The indicator function $\delta_{\phi}$ chooses the orientation of the road by using the previous motion estimate to resolve the heading ambiguity. Equation (15) improves the robustness of the filter because (i) it allows targets to cross roads without following the road and (ii) it can emphasize the constraints on some roads (e.g., bridges) more than the others.

Note that the mean $\phi_{\{\theta \text {, road }\}}$ of the heading prior is the orientation of the road, where the line, originating from the node with a slope angle $\theta$, intersects the road. Finally, in the weighting method, the heading prior enters the particle filter at the weighting stage as an independent pseudo-observation: $w_{t}^{*}{ }_{t}^{(i)}=$

$$
w_{t-T}^{(i)} \frac{p\left(\mathbf{y}_{t} \mid \mathbf{x}_{t}^{(i)}\right) p\left(\mathbf{x}_{t}^{(i)} \mid \mathbf{x}_{t-T}\right)}{\prod_{k} g_{k}\left(x_{k}^{(i)}(t) \mid \mathbf{y}_{t}, x_{k}^{(i)}(t-T)\right)} \prod_{k} p_{\left\{\theta_{k}^{(i)}, \text { road }\right\}}\left(\phi_{k}^{(i)}\right) .
$$

\section{The Mixture Method}

In $[1,3,4,6]$, the road prior information is used to change the state update equation to improve the predicted state values. For example, [4] uses Bayesian arguments with hospitability maps to fuse the road's preferred heading and speed values into the state update by the Ito stochastic differential equations. [3] also approaches the problem with Bayesian arguments and derives approximate closed-form solutions for the predicted state. [6] modifies the state noise variances so that the state noise aligns itself with the road for better prediction. [1] uses a similar approach using the interacting multiple model filter (IMM). 
The mixture method presented here does not try to modify the state update by using the road information. This is because the filter proposal function is based on an approximation of the motion posterior (interaction of the constant velocity and image observation models) and is independent from the road constraints. Instead, the mixture method tries to select the state update by monitoring the target motion in an exponentially decaying window. By adaptively varying the mixture components based on the data, the mixture method results in improved state estimates, while adaptively identifying the road branch.

To demonstrate the mixture method, we first modify the state update equation of the particle filter as follows:

$p\left(x_{k}(t) \mid x_{k}(t-T), \mathbf{m}_{t}^{\{k\}}\right)=\sum_{j=0}^{J_{t}-1} m_{j, t}^{\{k\}} f_{j}\left(x_{k}(t) \mid x_{k}(t-T)\right)$,

where $J_{t}$ is the number of road branches at DOA $\theta_{k}(t)$, $\mathbf{m}_{t}^{\{k\}}=\left[m_{0, t}^{\{k\}}, \ldots, m_{J-1, t}^{\{k\}}\right]$, where $m_{j, t}^{\{k\}}$ are the mixture probabilities for target $k$; and $f_{j}\left(x_{k}(t) \mid x_{k}(t-T)\right)$ are the state update functions. That is, $f_{0}\left(x_{k}(t) \mid x_{k}(t-T)\right)=$ $\mathcal{N}\left(x_{k}(t), \Sigma_{u}\right)$ handles the case when the target is stationary, $f_{1}\left(x_{k}(t) \mid x_{k}(t-T)\right)$, given by (3), handles the case when the target is moving with constant velocity; and $f_{j}\left(x_{k}(t) \mid x_{k}(t-\right.$ $T))$ is the state update based on the heading of the $(j-1)^{\text {th }}$ road branch.

To calculate $f_{j}\left(x_{k}(t) \mid x_{k}(t-T)\right)$, we propagate the particle set $x_{k}^{(i)}(t-T)$ through the state update function $\tilde{x}_{k}^{(i)}(t)=$ $h_{T}\left(x_{k}^{(i)}(t-T)\right)$. Then, the DOA of $\tilde{x}_{k}^{(i)}(t)$ is used to determine the heading of the road $\phi_{\left\{\tilde{\theta}^{(i)}, \operatorname{road}(j)\right\}}(t)$. We replace the heading parameters of $x_{k}^{(i)}(t-T)$ with new realizations $\phi_{k}^{(i)}(t-T) \sim \mathcal{N}\left(\phi_{\left\{\tilde{\theta}^{(i)}, \operatorname{road}(j)\right\}}(t), \sigma_{\phi, \operatorname{road}(j)}^{2}\right)$ to obtain $\hat{x}_{k}^{(i)}(t-T)$. Given this augmented state based on the road heading information, the $j^{\text {th }}$ road branch state update function is given by $f_{j}\left(x_{k}^{(i)}(t) \mid x_{k}^{(i)}(t-T)\right)=$ $\mathcal{N}\left(h_{T}\left(\hat{x}_{k}^{(i)}(t-T)\right), \Sigma_{u}\right)$.

To determine the mixture parameters, we use an EM algorithm for mixture models $[18,32,33]$. We consider the temporal state estimates under an exponential envelope $\epsilon_{t}(l)=$ $\beta \exp \left(-\frac{t-l}{\rho}\right)$, where $l \leq t$. The parameter $\rho$ is the half-life of the envelope, and $\beta=1-\exp \left\{-\rho^{-1}\right\}$ is the normalizing constant. The loglikelihood of the state evolution up to time $t$ can be written as

$L\left(\mathbf{X}_{t}(k) \mid \mathbf{M}_{t}\right)=\sum_{l=-\infty}^{t} \epsilon_{t}(k) \log p\left(x_{k}(l) \mid x_{k}(l-1), \mathbf{m}_{l}^{\{k\}}\right)$

where $\mathbf{X}_{t}(k)=\left[x_{k}(t), x_{k}(t-1), \ldots\right]$ and $\mathbf{M}_{t}=$ $\left[\mathbf{m}_{t}^{\{k\}}, \mathbf{m}_{t-1}^{\{k\}}, \ldots\right]$.

Given previous time estimates for the mixture probabilities in the particle filter, the E-step of the EM algorithm calculates the ownership probabilities for each new state $x_{k}(t)$ for each particle $i$ :

$$
o_{j, t}^{i}(k) \propto \frac{m_{j, t-1}^{\{k\}} f_{j}\left(x_{k}^{(i)}(t) \mid x_{k}^{(i)}(t-1)\right)}{p\left(x_{k}^{(i)}(t) \mid x_{k}^{(i)}(t-1), \mathbf{m}_{t}^{\{k\}}\right)} .
$$

Conditioned on the ownership probabilities, the M-step computes the mixture probabilities for the current time step:

$$
m_{j, t}^{\{k\}}(i)=\sum_{l=-\infty}^{t} \epsilon_{t}(k) o_{j, t}^{i}(k)
$$

Since it is not possible to store the whole temporal state evolution, we approximate the mixture probability calculation by a simple weighted average:

$$
m_{j, t}^{\{k\}}(i)=\beta o_{j, t}^{i}(k)+(1-\beta) m_{j, t-1}^{\{k\}}(i) .
$$

Hence, the particle filter first proposes particles according to the full posterior approximation, independent from the road information. Then, by calculating the current mixture probabilities, it determines the current state update model:

$p\left(x_{k}^{(i)}(t) \mid x_{k}^{(i)}(t-1)\right)=f_{\left\{\arg \max _{j} m_{j, t}^{\{k\}}(i)\right\}}\left(x_{k}(t) \mid x_{k}(t-T)\right)$.

In turn, the weighting step weights the particles according to the chosen state update model:

$$
w_{t}^{*}{ }_{t}^{(i)}=w_{t-T}^{(i)} \frac{p\left(\mathbf{y}_{t} \mid \mathbf{x}_{t}^{(i)}\right) \prod_{k} p\left(x_{k}^{(i)}(t) \mid x_{k}^{(i)}(t-T)\right)}{\prod_{k} g_{k}\left(x_{k}^{(i)}(t) \mid \mathbf{y}_{t}, x_{k}^{(i)}(t-T)\right)} .
$$

\section{Simulations}

In this section, we demonstrate the performance of the DOAonly particle filter with a synthetic example. We use an oval track shown in the bottom left subplot in Figs. 2, 3, 4, and 5 that has a width of $100 \mathrm{~m}$. The oval track is segmented into three segments to simulate road branching: the first segment from $(-100,-50) \mathrm{m}$ to $(0,-50) \mathrm{m}$, the second segment the top part of the oval, and the third segment is from $(0,-50) \mathrm{m}$ to $(150,-50) \mathrm{m}$. The simulation parameters in this section are summarized in Table 1 . Note that we also create an estimated target track in the $x-y$ space by using the filter motion estimates along with the correct initial target position. In the simulation figures, the particle filter with no road prior information is marked with $(\cdot)$, the weighting method is marked with $(+)$, and the mixture method is marked with (o).

In Fig. 2, we compare the tracking performance of the particle filter with and without the presented road prior methods. The target starts at $(-100,-50) \mathrm{m}$ and travels on the oval path for a duration of 30 seconds with a speed of $14 \mathrm{~m} / \mathrm{s}$. In this simulation, we use $\sigma_{\phi, \operatorname{road}(j)}^{2}=\left(5^{\circ}\right)^{2}$ for the weighting method. Among the mixture method and the weighting method, the weighting method performs the best in this case. Both methods improve the target state estimates over the particle filter, because they use the road information. In addition to the 
Table 2. Simulation Parameters

\begin{tabular}{||c|c||}
\hline \hline Number of particles, $N$ & 400 \\
\hline$\theta$ noise $\sigma_{\theta, k}$ & $1^{\circ}$ \\
\hline Q noise $\sigma_{Q, k}$ & $0.05 \mathrm{~s}^{-1}$ \\
\hline$\phi$ noise $\sigma_{\phi, k}$ & $10^{\circ}$ \\
\hline Measurement noise $\sigma_{\theta}$ & $1^{\circ}$ \\
\hline Road prior heading variance $\sigma_{\phi, \text { road }}$ & $5^{\circ}$ \\
\hline Tracker sampling period, $\mathrm{T}$ & $1 \mathrm{~s}$ \\
\hline Beamformer batch period, $\tau$ & $0.1 \mathrm{~s}$ \\
\hline Clutter density parameter, $\lambda$ & 600 \\
\hline Probability of target miss $\kappa_{0, K}^{f}$ & 0.1 \\
\hline Envelope half-life $\rho$ & $1 \mathrm{~s}$ \\
\hline Number of batch samples for DOA estimation, $\mathrm{M}$ & 10 \\
\hline Number of DOA peaks in the beamformer pattern $P$ & 2 or 4 \\
\hline Narrow-band beamformers at different center frequencies, $\mathrm{F}$ & 1 \\
\hline \hline
\end{tabular}

tracking results, the mixture method probabilities, shown in Fig. 2(b), intuitively tell that the target starts at the first segment and then travels to the second segment.

In Fig. 3, we compare the state tracking performances when a target comes off the road. In this case, the mixture model performs the best, followed by the weighting method and the particle filter with no road prior information. In Fig. 4, we demonstrate the algorithms for a multi target scenario. Lastly, we show the effect of the road heading variance parameter $\sigma_{\phi, \operatorname{road}(j)}^{2}$ in Fig. 5. When the road heading variance is small $\sigma_{\phi, \text { road }}^{2}=\left(2^{\circ}\right)^{2}$, the resulting track is tighter.

\section{CONCLUSIONS}

In this paper, we proposed two methods to incorporate prior road information into a DOA-only multi target tracking particle filter. The weighting method treats the road information as an additional independent observation on the target heading direction. The mixture method incorporates the road information into the state update by using probability mixtures. By selecting the model using an online EM algorithm, the mixture model improves the state estimates, while simultaneously resulting in a temporal probability track for road segments. The presented methods improve the state vector estimates when the targets are following the road constraints; however, they do not degrade the performance if the targets do not follow the available road network.

\section{REFERENCES}

[1] T. Kirubarajan, Y. Bar-Shalom, K.R. Pattipati, I. Kadar, B. Abrams, and E. Eadan, "Tracking ground targets with road constraints using an IMM estimator," in Proceedings of the IEEE Aerospace Conference, 21-28 March 1998, vol. 5.

[2] S. Gattein and P. Vannoorenberghe, "A comparative analysis of two approaches using the road network for tracking ground targets," in Proceedings of the Seventh International Conference on Information Fusion, Stockholm, Sweden, 28 June-1 July 2004.
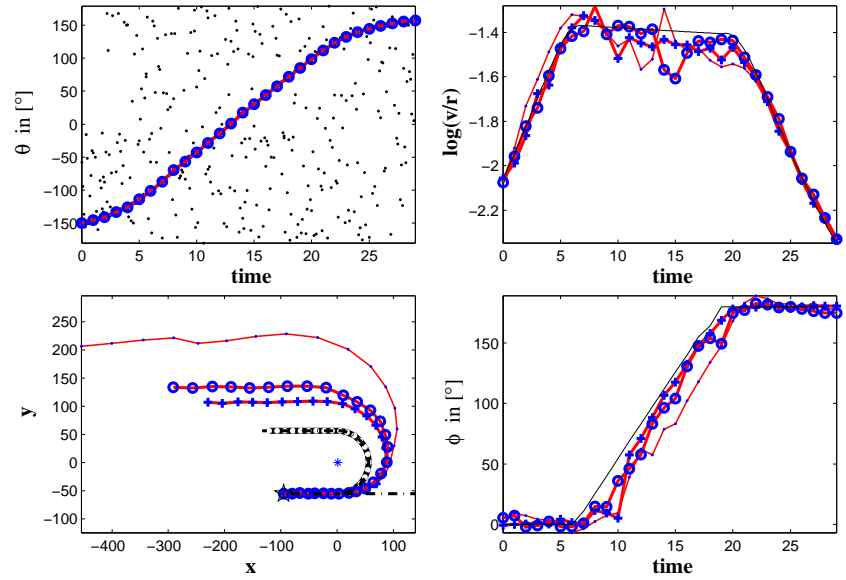

(a)

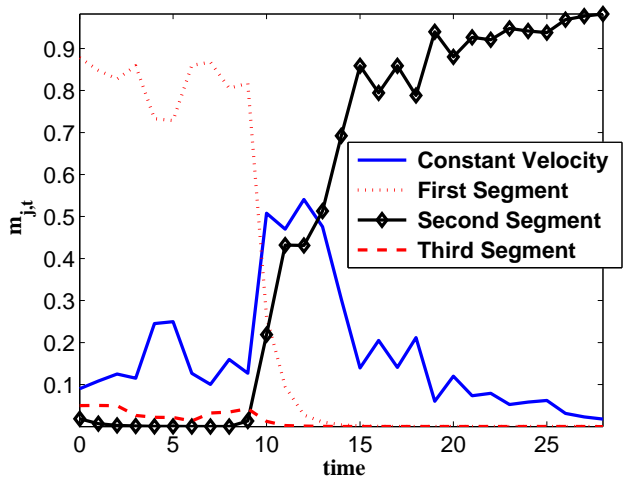

(b)

Figure 2. (a) The DOA tracking estimates of the particle filter tracker with and without the road prior information are similar. However, the velocity-over-range and the heading estimates improve due to the constrained target path. Note that the particle filter heading estimates without the road information lag the true target heading due to the constant velocity assumption. (b) The mixture probabilities coming from the mixture method shows the temporal likelihood of the paths taken by the target.

[3] R.H. Enders, "Fundamentals of on-road tracking," in SPIE Conference on Acquisition, Tracking, and Pointing XIII, Orlando, Florida, April 1999, vol. 3692.

[4] K. Kastella, C. Kreucher, and M.A. Pagels, "Nonlinear filtering for ground target applications," in Proceedings of SPIE, 2000, vol. 4048.

[5] P. Day and P. Bladon, "Using prior information to enhance tracking," in SPIE Conference on Acquisition, Tracking, and Pointing XVI, Bellingham, WA, 2004, vol. 5430 .

[6] B. Pannetier, K. Benameur, V. Nimier, and M. Rombaut, "Ground moving target tracking with road constraint," in SPIE Conference on Signal Processing, Sensor Fusion, and Target Recognition XIII, Bellingham, WA, 2004, vol. 5429. 

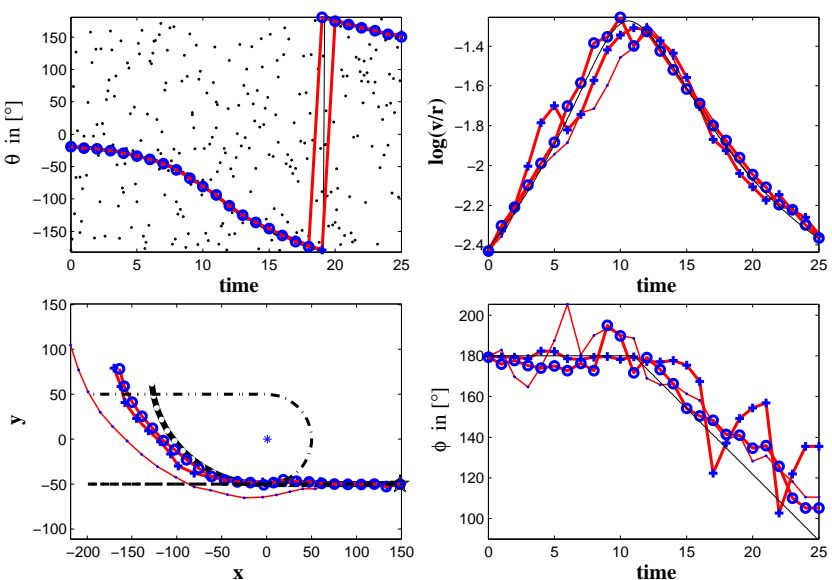

Figure 3. The formulations of the weighting and mixture methods allow the targets to wander off-road. When the prediction model of the particle filter proposes particles based on the road only, the particle filter tracker either diverges or generates a shadow track on the straight segment.
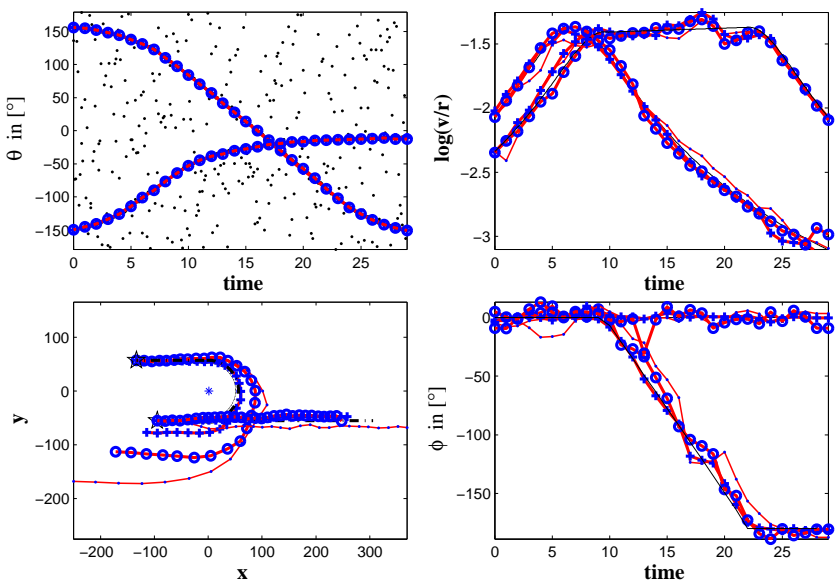

Figure 4. Multi target tracking scenario. The filter does not have any association problems when the target DOA's cross at $t=17 \mathrm{~s}$ because of the other motion state variables.
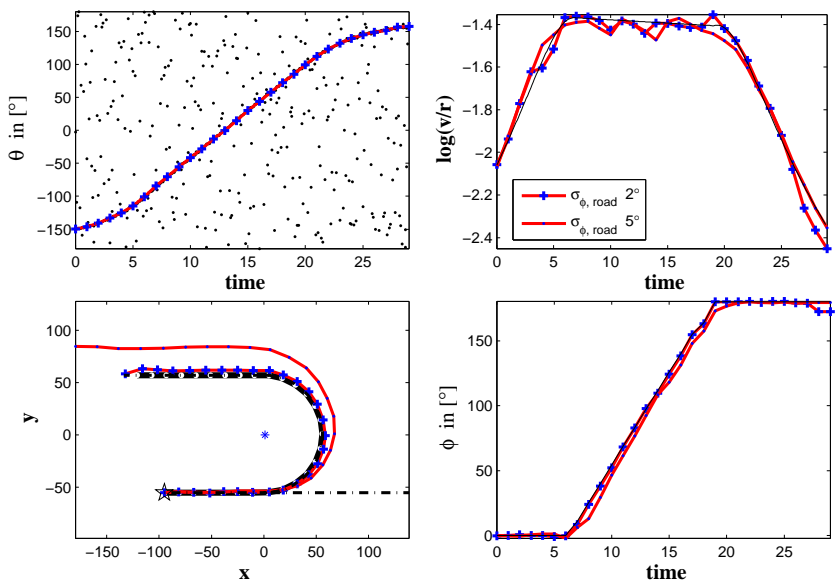

Figure 5. The effect of the road heading variance parameter $\sigma_{\phi, \operatorname{road}(j)}^{2}$ is demonstrated.
[7] C.K. Sword, M. Simaan, and E.W. Kamen, "Multiple target angle tracking using sensor array output," IEEE Trans. Aerosp. Electron. Syst., vol. 26, pp. 367-372, Mar. 1990.

[8] M. Orton and W. Fitzgerald, "A Bayesian approach to tracking multiple targets using sensor arrays and particle filters," IEEE Trans. on Signal Processing, vol. 50, no. 2, pp. 216-223, February 2002.

[9] Y. Zhou, P.C. Yip, and H. Leung, "Tracking the direction-of-arrival of multiple moving targets by passive arrays: Algorithm," IEEE Trans. on Signal Processing, vol. 47, no. 10, pp. 2655-2666, October 1999.

[10] V. Cevher and J. H. McClellan, "General directionof-arrival tracking with acoustic nodes," IEEE Trans. on Signal Processing, vol. 53, no. 1, pp. 1-12, January 2005.

[11] J. R. Larocque, J. P. Reilly, and W. Ng, "Particle filters for tracking an unknown number of sources," IEEE Trans. on Signal Processing, vol. 50, no. 12, pp. 29262937, Dec. 2002.

[12] T. Kailath, A.H. Sayed, and B. Hassibi, Linear Estimation, Prentice Hall, 2000.

[13] W.L. Brogan, Modern Control Theory, Prentice Hall, 1991.

[14] A. Doucet, N. Freitas, and N. Gordon, Eds., Sequential Monte Carlo Methods in Practice, Springer-Verlag, 2001.

[15] J.S. Liu and R. Chen, "Sequential Monte Carlo methods for dynamic systems," Journal of the American Statistical Association, vol. 93, pp. 1032-1044, September 1998.

[16] V. Cevher and J. H. McClellan, "Acoustic direction-ofarrival multi target tracking," submitted to IEEE Transactions on Signal Processing.

[17] Y. Bar-Shalom and T. Fortmann, Tracking and Data Association, Academic-Press, 1988.

[18] R. A. Redner and H. F. Walker, "Mixture densities, maximum likelihood and the EM algorithm," SIAM Review, vol. 26, pp. 195-239, April 1984.

[19] A. P. Dempster, N. M. Laird, and D. B. Rubin, "Maximum likelihood from incomplete data via the EM algorithm," Journal of Royal Statistical Society, Series B, vol. 39, pp. 1-38, 1977.

[20] H.V. Poor, An Introduction to Signal Detection and Estimation, Springer-Verlag, 1994.

[21] L. Tierney and J. B. Kadane, "Accurate approximations for posterior moments and marginal densities," Journal of the American Statistical Association, , no. 81, pp. 82$86,1986$.

[22] A. Gelman, J. B. Carlin, H. S. Stern, and D. B. Rubin, Bayesian Data Analysis, Chapman Hall/CRC, 2004. 
[23] D.H. Johnson and D.E. Dudgeon, Array Signal Processing: Concepts and Techniques, Prentice Hall, 1993.

[24] A. Papoulis and S.U. Pillai, Probability, random variables and stochastic processes, McGraw Hill, 2002.

[25] J. MacCormick and A. Blake, "A probabilistic exclusion principle for tracking multiple objects," in $7^{\text {th }}$ International Conference on Computer Vision, 1999, pp. 572-578.

[26] M. Evans, N. Hastings, and B. Peacock, Statistical Distributions, 3rd ed., Wiley, 2000.

[27] T. Edgoose, L. Allison, and D. L. Dowe, "An MML classification of protein sequences that knows about angles and sequences," in Pacific Symp. Biocomputing 98, Jan. 1998, pp. 585-596.

[28] Z. Khan, T. Balch, and F. Dellaert, "An MCMC-based particle filter for tracking multiple interacting targets," Tech. Rep. GIT-GVU-03-35, College of Computing, Georgia Institute of Technology, Oct. 2003.

[29] V. Cevher and J. H. McClellan, "An acoustic multiple target tracker," in IEEE SSP 2005, Bordeaux, FR, 17-20 July 2005.

[30] J.E.Dennis Jr. and R.B. Schnabel, Numerical Methods for Unconstrained Optimization and Nonlinear Equations, Society for Industrial and Applied Mathematics, Philadelphia, PA, 1996.

[31] J. Nocedal and S.J. Wright, Numercial Optimization, Springer-Verlag, 1999.

[32] A.D. Jepson, D.J. Fleet, and T. El-Maraghi, "Robust online appearance model for visual tracking," IEEE Trans. on Pattern Anal. and Mach. Int., vol. 25, pp. 1296-1311, Oct. 1998.

[33] S.K. Zhou, R. Chellappa, and B. Moghaddam, "Visual tracking and recognition using appearance-adaptive models in particle filters," IEEE Trans. Image Processing, vol. 13, pp. 1491-1506, November 2004.

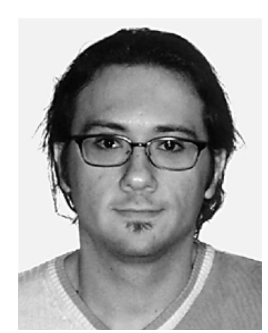

Volkan Cevher was born in Ankara, Turkey, in 1978. He received his B.S. degree in Electrical Engineering from Bilkent University, Ankara, Turkey in 1999 as a valedictorian. During summer of 2003, he was employed by Schlumberger Doll Research. In Fall 2004, he was the co-recipient of the Center for Signal and Image Processing Outstanding Research Award. He received his Ph.D. degree in Electrical Engineering from Georgia Institute of Technology in 2005. He worked a post-doc at Georgia Institute of Technology under the supervision of Dr. James H. McClellan till the end of 2005. His currently working with Dr. Rama Chellappa as a research associate on image processing problems. His research interests include Monte-Carlo Markov chain methods (specifically particle filters), target tracking models, adaptive filters, timefrequency distributions, fractional Fourier transform, and array signal processing.

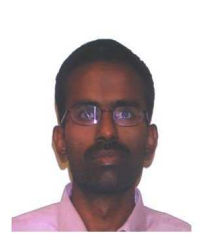

Rajbabu Velmurugan received the B.E. degree from Government College of Technology, Coimbatore, India in 1995 and the M.S. degree from Clarkson University, Potsdam, USA in 1998, both in electrical engineering. He worked in Larsen \& Toubro Ltd., India before joining Clarkson University and in The MathWorks from 1998 to 2001. He is currently pursuing his Ph.D. degree at Georgia Institute of Technology under the advisement of Prof.James H McClellan. His research interests include array signal processing, real-time signal processing, and particle filtering techniques. He is interested in teaching and involved in educational projects related to signal processing.

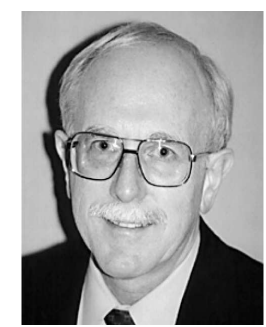

James $\boldsymbol{H}$. McClellan received the B.S. degree in Electrical Engineering from L.S.U. in 1969, and the M.S. and Ph.D. degrees from Rice University in 1972 and 1973, respectively. From 1973 to 1982, he was a member of the research staff at Lincoln Laboratory and then a professor at MIT. From 1982 to 1987, Dr. McClellan was employed by Schlumberger Well Services. Since 1987, he has been a Professor in the School of Electrical and Computer Engineering at Georgia Tech, where he presently holds the Byers' Professorship in Signal Processing.

He is a co-author of the texts Number Theory in Digital Signal Processing, Computer Exercises for Signal Processing, DSP First: A Multimedia Approach, and Signal Processing First, which received the McGraw-Hill Jacob Millman award for an innovative textbook in 2003. In 1998, Prof. McClellan received the W. Howard Ector Outstanding Teacher Award at Georgia Tech, and in 2001, the Education Award from the IEEE Signal Processing Society. In 1987, he received the Technical Achievement Award for work on FIR filter design, and in 1996, the Society Award, both from the IEEE Signal Processing Society. In 2004, he was a co-recipient of the IEEE Kilby medal. Prof. McClellan is a Fellow of the IEEE and a member of Tau Beta Pi and Eta Kappa Nu. 